\title{
Physikalische Therapie: Therapiemittel Licht - ausgewählte Themen
}

Jean-Michel Jeannin

Basel, Schweiz

\section{Einleitung}

Die Haut, namentlich die Gesichtshaut, ist ein essenzielles Ausdrucksorgan des Menschen. Chronische Erkrankungen der Haut wie die atopische Dermatitis oder die Lichtdermatosen sind zwar nicht lebensbedrohlich, aber beeinträchtigen die Lebensqualität und die soziale Integration der Betroffenen in erheblichem Masse. Der vorliegende Beitrag behandelt die Möglichkeiten der Phototherapie und Klimatherapie zur Behandlung dieser Krankheiten anhand ausgewählter Beispiele (Abb. 1).

\section{Atopische Dermatitis}

Die atopische Dermatitis (endogenes Ekzem) ist eine chronische Entzündung der Haut und wird durch starken Juckreiz sowie rekurrierende Ekzeme charakterisiert (Abb. 2). In entwickelten Ländern sind 20\% der Kinder und $10-20 \%$ der Gesamtbevölkerung betroffen. Die atopische Dermatitis kann für die Betroffenen und ihre An-

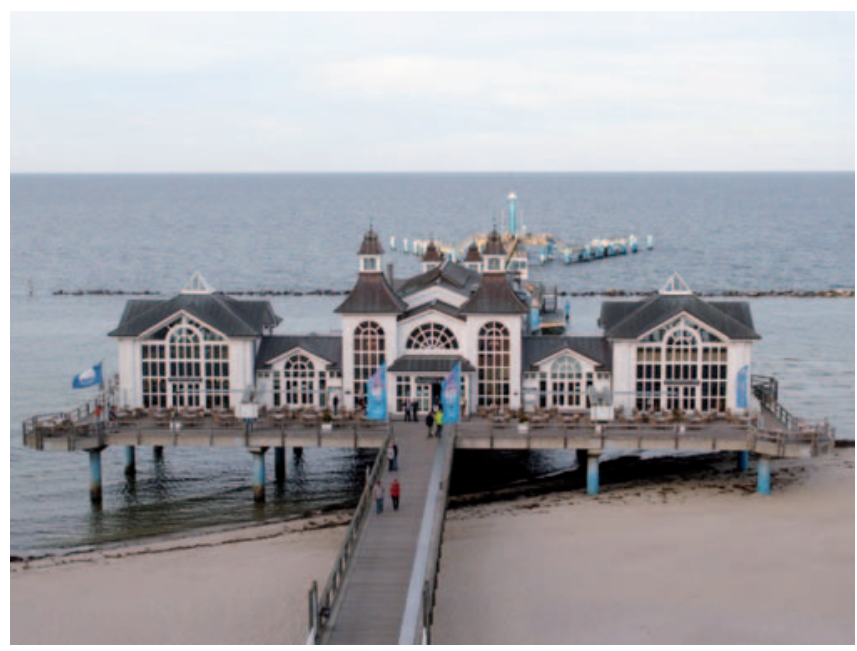

Abb. 1. Ostseebad Sellin. gehörigen psychisch und sozial sehr belastend sein. $\mathrm{Zu}$ dem ist das Risiko für eine Nahrungsmittelallergie, für Asthma, für eine allergische Rhinitis sowie für psychische Krankheiten erhöht. Gemäss aktuellstem Stand des Wissens handelt es sich bei der atopischen Dermatitis um eine lebenslange Veranlagung mit einer Störung der Barrierefunktion der Haut als zentralem pathogenetischen Faktor: Die Haut der Betroffenen wehrt Antigene und Keime weniger gut ab als die gesunde Haut [1]. Allergien und Infektionen (vornehmlich durch Staphylococcus aureus) sind häufige Folgen [1]. Die Veranlagung ist erblich. Erbgang und betroffene Gene sind derzeit noch unklar [2]. Im Ganzen wird die Krankheit noch wenig verstanden [1].

In $85 \%$ der Fälle manifestiert sich die Krankheit in den ersten 5 Lebensjahren und bildet sich in $40 \%$ der Fälle bis zum Erwachsenenalter zurück [2]. Die atopische Dermatitis gilt jedoch derzeit als nicht heilbar [1].

Die Grundbehandlung besteht in der Verabreichung von Präparaten, welche die Haut feucht halten und fetten. Exantheme werden mit Kortikosteroiden topisch sowie mit verschiedenen Modalitäten der Phototherapie und mit der physikalischen Komplextherapie behandelt. Systemisch werden Cyclosporine, Azathioprin, Mycophenolatmofetil und Methotrexat verabreicht [3]. Phytotherapeutisch können Johanniskrautöl lokal [4] sowie Nachtkerzenöl peroral [5] mit Erfolg angewendet werden (Abb. 3).

21 Patienten, die an einer schweren atopischen Dermatitis litten und stark wirksame Kortikosteroide benötigten, wurden nach 12 Wochen bisheriger Therapie während 12 Wochen dreimal pro Woche mit kurzwelligem UVBLicht behandelt und anschliessend noch 24 Wochen beobachtet (Follow-up). Am Ende der Behandlungsphase war der Schweregrad im Urteil des Prüfarztes und im Urteil der Patienten um 68\% sowie der Gebrauch von Kortikosteroiden um $80 \%$ zurückgegangen. Am Ende der Follow-up-Periode war bei 6 Patienten der Schweregrad wieder auf dem Wert vor der Therapie. Die übrigen 15 Patienten benötigten weiterhin weniger Kortikosteroide.

\section{KARGER}

Fax +497614520714 Information@Karger.com www.karger.com

\section{() 2016 S. Karger GmbH, Freiburg}




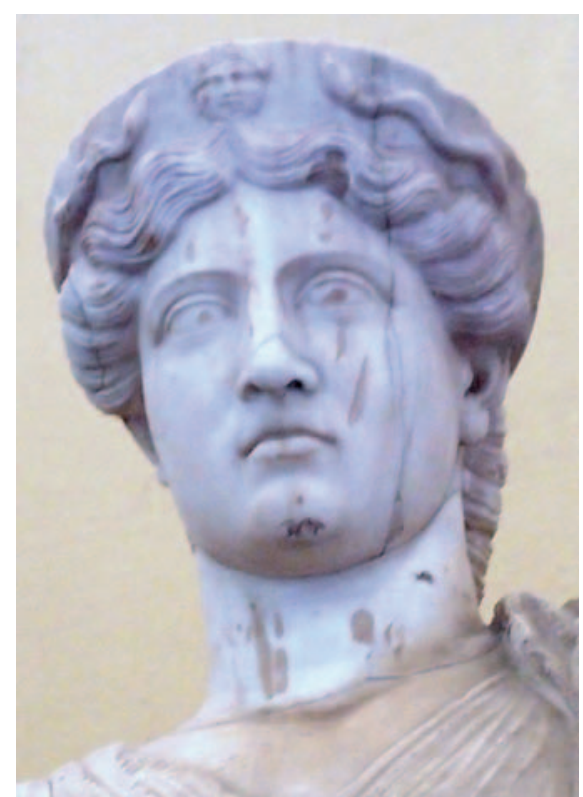

Abb. 2. Beschädigte «Haut» einer Statue im Vatikan.

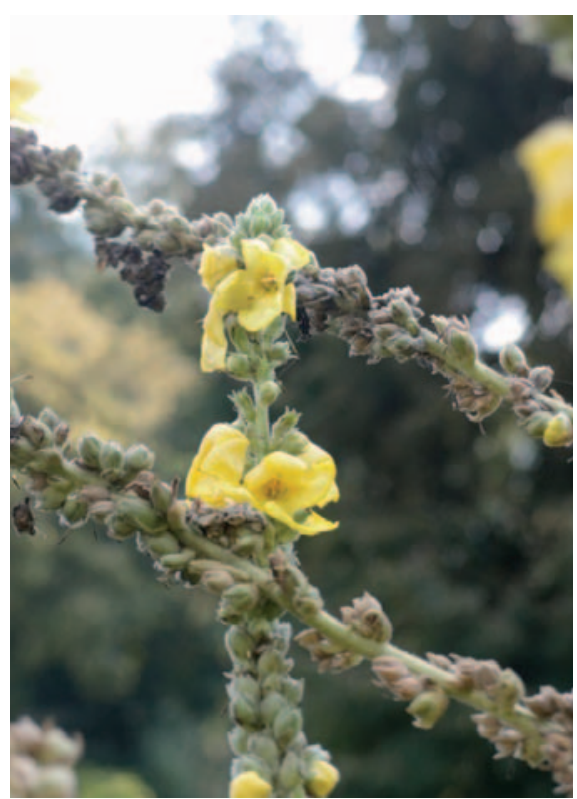

Abb. 3. Nachtkerze (Oenothera).
Die meisten Patienten hatten im Verlauf der Behandlung eine Rötung der Haut. Keine der unerwünschten Wirkungen führte zu einem Behandlungsabbruch [6].

In einer Vergleichsstudie wurde die Wirksamkeit einer UVA-, einer niedrig dosierten UVB- und einer kombinierten UVA-UVB-Phototherapie verglichen. Die Wirksamkeit wurde anhand der Variablen Juckreiz, Lichenifizierung, Schuppung, Trockenheit, Bläschenbildung, Exkoriationen (oberflächliche Hautdefekte) und Rötung sowie anhand der Gesamtauswertung bestimmt. Als Ergebnis wurde die Wirksamkeit einer kombinierten Phototherapie am besten beurteilt. Kritisch fügen die Autoren an, dass UVA-Lampen auch UVB-Licht und UVB-Lampen auch UVA-Licht ausstrahlen, was die Beurteilung verkompliziert. Die Wirkungsweise einer Phototherapie mit UV-Licht ist im Einzelnen nicht geklärt. An der Wirkung beteiligt sind immunologische, antimikrobielle und die Epidermis verstärkende Eigenschaften [7].

Eine vierwöchige Kur am Toten Meer für 27 Patienten unter 19 Jahren mit einer atopischen Dermatitis vermochte den Atopic Dermatitis Index um 87,5 $\pm 13,4 \%$ bei Abschluss der Kur und 3 Monate später beim Follow-up um $71,3 \pm 21,3 \%$ zu verbessern. Der Index wurde beim einzelnen Patienten jeweils vom selben Dermatologen beurteilt [8].

\section{Lichtdermatosen (Photodermatosen)}

Lichtdermatosen sind abnorme Reaktionen der Haut auf UVA-Strahlung. Davon abzutrennen sind akute Reaktionen auf eine zu hohe UV-Bestrahlung. Primäre Lichtdermatosen werden durch photosensibilisierende
Substanzen ausgelöst. Bei sekundären Lichtdermatosen liegt eine internistische Grundkrankheit vor (z.B. Lupus erythematodes, Porphyrie, Xeroderma pigmentosum). Primäre Lichtdermatosen sind entweder idiopathisch (z.B. Lichturtikaria, polymorphe Lichtdermatose) oder durch chemische Photosensibilisierung verursacht. UVDosen, die üblicherweise gut vertragen werden, können in Verbindung mit exogenen oder endogenen photosensibilisierenden Substanzen zu Sonnenbrand-ähnlichen Reaktionen führen [9]. Exogene sensibilisierende Substanzen sind Phenothiazine (unter anderem Antihistaminika!), Furosemid, Furocumarine (z.B. aus Pflanzen) und Ciprofloxacin [10]; endogene sensibilisierende Substanzen sind z.B. Porphyrine. Phototoxische Hautreaktionen treten häufiger auf als photoallergische. Sie beruhen auf einer Reaktion auf Wiesengräser (Dermatitis pratensis) oder z.B. auf Tetracycline. Die polymorphe Lichtdermatose («Sonnenallergie») ist die häufigste Lichtdermatose (Prävalenz in Mitteleuropa, Skandinavien und den USA: 10-20\%). Sie tritt in den Monaten März bis Juni auf und kann jedes Lebensalter, auch das Kindesalter, betreffen. Frauen sind neunmal häufiger als Männer betroffen [9]. Die Hauterscheinungen bilden sich nach Beendigung der Exposition rasch zurück. Unterstützend können Kortikosteroide verabreicht werden. Zur Behandlung des Juckreizes eignen sich Antihistaminika innerlich und lokal [9]. Phototherapeutische Behandlungen gelangen hauptsächlich zur Prophylaxe zum Einsatz [9]. Die Lichturtikaria (Urticaria solaris) ist eine schwere Erkrankung und stellt $0,08 \%$ aller Urtikaria-Fälle dar. Es wird zwischen einer UVB-sensiblen und UVB-insensiblen Lichturtikaria unterschieden [11]. Kurzwelliges UVB-Licht ist zur Prophylaxe gut wirksam [10]. 


\section{Reflexionen}

Die atopische Dermatitis ist eine vielfältige Krankheit mit einer stark individuellen Ausprägung. Trotz ihrer Häufigkeit wird sie nur wenig verstanden. Die Standardtherapie mit der Langzeitanwendung von Kortikosteroiden ist mit lokalen (unter anderem allergische Reaktionen) und systemischen Nebenwirkungen belastet [7]. Anhand der atopischen Dermatitis lässt sich ein Dilemma der orthodoxen Schulmedizin gut exemplifizieren: Die Behandlung mit Immunsuppressiva (z.B. Cyclosporin) wird als rationale («vernünftige») Therapie betrachtet, eine Kur am Toten Meer jedoch als unwirksam und nicht gerechtfertigt. Der Nachweis der Wirksamkeit einer individuellen Therapie, wie sie für die physikalische Therapie typisch ist, ist nach den üblichen Kriterien praktisch nicht möglich: Es fehlt ein Placebo bzw. eine Vergleichsbehandlung (4 Wochen täglich ein paar Stunden an der örtlichen Kreuzung liegen und das Klima einwirken lassen vs. 4 Wochen am Toten Meer liegen und das Klima einwirken lassen?). Ein mögliches Kriterium wäre die Berücksichtigung des individuellen Anpassungsgrades einer Therapie, wodurch jedoch ein Problem aufgrund der geringen Fallzahlen entsteht. Es ist unbestreitbar richtig, dass medika- mentöse Therapien nach strengsten Kriterien unter Beweis gestellt werden müssen: Der Verkauf eines unwirksamen Medikaments ist ethisch nicht vertretbar. Die Wirksamkeit von Immunsuppressiva beispielsweise lässt sich gut beweisen: Der Hautausschlag des Patienten bildet sich zurück. Was ist aber damit für den Patienten gewonnen? Eine physikalische Therapie behandelt mehr als nur die Hautausschläge. Vor allem beinhaltet sie sehr viel mehr Zuwendung. Eine Komplextherapie schliesst unter Umständen auch die Anwendung von schulmedizinischen Präparaten ein, ist naturgemäss aber breiter aufgestellt.

Die Lichtdermatosen offenbaren ein weiteres Dilemma der aktuellen Medizin: Primäre Lichtdermatosen werden unter anderem von bestimmten Medikamenten verursacht und durch natürliches Licht ausgelöst. Sie können aber auch medikamentös behandelt werden. Eine Phototherapie gilt als wirksame Prophylaxe und scheint im vorliegenden Zusammenhang anerkannt.

Fazit: Die Kriterien, die für den Nachweis der Wirksamkeit eines Medikaments gelten, sind nicht ohne Weiteres auf den Nachweis der Wirksamkeit einer komplexen Therapie anwendbar.

\section{Literatur}

1 Weidinger S, Novak N: Atopic dermatitis. Lancet 2016;387:1109-1122.

2 Rudikoff D, Lebwohl M: Atopic dermatitis. Lancet 1998;351:1715-1721.

3 Werfel T, et al: The diagnosis and graded therapy of atopic dermatitis. Dtsch Arztebl Int 2014;111:509-520.

4 Schempp C, et al: Topical treatment of atopic dermatitis with St. John's wort cream - a randomized, placebo controlled, double-blind half side comparison. Phytomedicine 2003;10 (suppl 4):31-37.

5 Bachmann C: Nachtkerzenöl zur Behandlung von atopischer Dermatitis. Ars Medici 2014;2: $448-451$.
6 George SA, et al: Narrow-band (TL-01) UVB air-conditioned phototherapy for chronic severe adult atopic dermatitis. $\mathrm{Br}$ J Dermatol 1993;128:49-56.

7 Jekler J, et al: Phototherapy for atopic dermatitis with ultraviolet A (UVA), low dose UVB and combined UVA and UVB: two pairedcomparison studies. Photodermatol Photoimmunol Photomed 1991;8:151-156.

8 Kudish A, et al: Dead Sea ultraviolet climatotherapy for children with atopic dermatitis. Photodermatol Photoimmunol Photomed 2016;DOI: 10.1111/phpp.12250.
Lehmann P, Schwarz T: Photodermatoses: diagnosis and treatment. Dtsch Arztebl Int 2001; 108:135-141.

10 Schauder S: Wenn Arzneimittel und Licht unverträglich sind. Pharmazeutische Zeitung online 2009; 18 .

11 Calzavara-Pinton P, et al: Narrowband ultraviolet $B$ phototherapy is a suitable treatment option for solar urticaria. J Am Acad Dermatol 2012;67:e5-9. 